Nota Científica

\title{
Avaliação de inseticida para controle da cigarra Quesada gigas em plantios de paricá
}

\author{
Odineila Martins Monteiro ${ }^{1}$, Alexandre Mehl Lunz², Roni de Azevedo², Moisés Mourão Júnior², Telma Fátima Vieira Batista ${ }^{1}$ \\ ${ }^{1}$ Universidade Federal Rural da Amazônia, Av. Tancredo Neves, 2501, Montese, CEP 66077-530, Belém, PA, Brasil

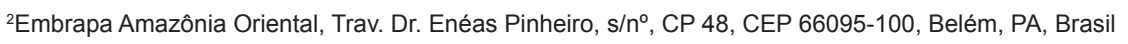

*Autor correspondente:
odineila.monteiro@gmail.com

Termos para indexação:

Praga florestal

Proteção de plantas

Schizolobium parahyba var. amazonicum

Cicadoidea

Hemiptera

Index terms:

Forest insect pest

Crop protection

Schizolobium parahyba var. amazonicum

Cicadoidea

Hemiptera

Histórico do artigo:

Recebido em 19/03/2014

Aprovado em 23/05/2014

Publicado em 07/07/2014

doi: 10.4336/2014.pfb.34.78.502
Resumo - Este trabalho objetivou determinar a dosagem mais eficiente e economicamente viável de inseticida químico para o controle de ninfas de Quesada gigas (Hemiptera: Cicadidae) em plantios de paricá. Foram testadas três dosagens de três produtos (carbofurano, imidacloprida e tiametoxam) baseadas na dosagem máxima recomendada para o controle de cigarras em cafeeiros e aplicadas em área total. A dosagem de um quilograma de produto comercial à base de tiametoxam por hectare foi a mais eficiente econômica e ambientalmente para controle de ninfas de Q. gigas em plantios de paricá.

\section{Evaluation of insecticides in different dosages to control cicadas in parica plantations}

\begin{abstract}
This study aimed to determine the more efficient and economically viable dosage of chemical insecticide to control Quesada gigas (Hemiptera: Cicadidae) nymphs in parica plantations. Three dosages of three products (carbofuran, imidacloprid and thiamethoxam) were tested based on the maximum recommended dosage for the control of cicadas in coffee plants and applied in total area. The dosage of one kilogram of a commercial product based in thiamethoxam per hectare was more efficient economically and environmentally to control nymphs of $Q$. gigas in parica plantations.
\end{abstract}

Aadaptação a sistemas produtivos puros e consorciados e as múltiplas utilidades de sua madeira tornaram o paricá, Schizolobium parahyba var. amazonicum (Huber ex Ducke) Barneby (Fabaceae), uma das essências florestais nativas mais cultivadas em todo o país. Com 87.900 ha cultivados no Pará, Maranhão e Tocantins (Anuário..., 2013), é espécie amplamente utilizada no setor produtivo madeireiro amazônico, com aplicação nos setores de laminados, compensados (Carvalho, 2006, 2007), painéis aglomerados (Iwakiri et al., 2010) e, mais recentemente, chapas de MDF (Medium-Density Fiberboard).
No Pará, a cigarra, Quesada gigas Olivier, 1790 (Hemiptera: Cicadidae), é a principal praga da cultura de paricá (Zanuncio et al., 2004; Lunz et al., 2010), que é severamente prejudicada pela incidência do inseto cuja ninfa subterrânea suga continuamente a seiva das raízes das plantas a ponto de comprometer talhões inteiros, dependendo da intensidade do ataque. Testes de eficiência de inseticidas registrados para a cultura do cafeeiro, onde existem quatro gêneros de cigarras de importância econômica (Martinelli \& Zucchi, 1997), apresentaram bons resultados em plantios de paricá, através de uma metodologia de avaliação com 
uso de uma grade modificada, que permite a contagem de galerias e ninfas de cigarras após a raspagem do solo (Lunz et al., 2010, 2012). Contudo, as dosagens empregadas naquela ocasião foram elevadas, restando definir quais seriam os inseticidas e dosagens mais eficazes, econômicos e com menos impacto ambiental.

O objetivo deste trabalho foi determinar a dosagem mais eficiente de diferentes inseticidas no controle de ninfas de $Q$. gigas em plantios de paricá.

O trabalho foi realizado de fevereiro a abril de 2010, na Fazenda Chapadão III, no Município de Dom Eliseu,

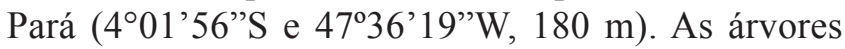
da área experimental possuíam seis anos de idade e faziam parte de uma área de cerca de 4.000 ha de plantios homogêneos de paricá com espaçamento de 4,0 $\mathrm{m}$ x 3,5 m. A vegetação de sub-bosque, onde as avaliações foram conduzidas, foi suprimida por roçada mecanizada para facilitar a contagem das ninfas de cigarras (Lunz et al., 2012).

As avaliações foram conduzidas segundo metodologia proposta por Lunz et al. (2010), onde o uso de uma grade aradora adaptada acoplada a um implemento tratorizado permitiu a abertura de trincheiras padronizadas, nas quais procedeu-se a contagem de ninfas de cigarras. Foi feita uma amostragem prévia, antes da aplicação dos inseticidas, seguida de cinco avaliações, aos 15, 30, 45, 60 e 75 dias após a aplicação, para verificação do poder residual dos inseticidas testados nos plantios de paricá. A cada amostragem eram abertas novas trincheiras, dado o fato de o método ser destrutivo. $\mathrm{O}$ delineamento experimental foi em blocos ao acaso, com dez tratamentos (três inseticidas em três dosagens mais o controle) e três repetições. A parcela experimental foi composta por 88 árvores $\left(980 \mathrm{~m}^{2}\right)$, onde foram abertas aleatoriamente três trincheiras de $0,8 \mathrm{~m}$ de largura, 0,07 $\mathrm{m}$ de profundidade e $7 \mathrm{~m}$ de comprimento cada, distribuídas em cada parcela. A área experimental total foi de 2,94 ha.

A aplicação dos inseticidas foi feita com um pulverizador tratorizado Mec Pec (Mecmaq Ltda., Piracicaba, Brasil), na área total de cada parcela, com volume de calda de $280 \mathrm{~L} \mathrm{x} \mathrm{ha}^{-1}$ e faixa de aplicação de $8 \mathrm{~m}$ de largura. A calda foi aplicada na linha passada pelo trator e nas metades das linhas adjacentes através de dois bicos voltados para o solo e um voltado para cada linha adjacente, fazendo com que o trator passasse alternadamente nas linhas de modo a aplicar os produtos em área total. Os inseticidas utilizados foram os mesmos empregados por Lunz et al. (2010), em menores quantidades, também usando-se como referência e dosagem máxima aquela registrada para controle de cigarras em cafezais: tiametoxam, nas dosagens de 1,0; 1,5 e $2,0 \mathrm{~kg} \mathrm{ha}^{-1}$ do produto comercial Actara (250 WG); carbofurano, com 4,0; 6,0 e 8,0 $\mathrm{L} \mathrm{ha}^{-1}$ de Ralzer (350 TS); e imidacloprida, com 2,5; 3,75 e 5,0 $\mathrm{L} \mathrm{ha}^{-1}$ de Provado (200 SC), sendo usada água no tratamento controle.

Os dados foram analisados com aplicação do modelo linear geral (GLM) e o valor de densidade da categoria de avaliação (ninfas das cigarras) foi transformado por arco seno de $(x+0,5)^{0,5}$. O modelo foi testado pelo teste $\mathrm{F}$ e os valores médios foram ordenados pelo teste de Duncan, a 5\% de probabilidade.

O efeito da aplicação dos inseticidas na dinâmica populacional de ninfas de cigarras foi medido através da proporção da diferença entre os valores iniciais e finais de insetos e ajustado mediante o uso de um polinômio de primeira ordem invertido expresso por:

$$
\mathrm{y}=\mathrm{y} 0+(\mathrm{a} / \mathrm{x})
$$

Onde: 'y’ é a proporção da redução da população, 'y0' e 'a' são coeficientes do modelo polinomial de primeira ordem inverso e ' $x$ ' é o número de ninfas na primeira observação.

O método de amostragem empregado indicou redução do número de ninfas para as dosagens dos inseticidas testados, ao se considerar o número médio acumulado de insetos observados no decorrer das avaliações (Figura 1). No entanto, a eficácia contínua no decorrer das avaliações foi evidenciada para os inseticidas tiametoxam e carbofurano, nesta ordem, sendo os resultados verificados com imidacloprida pouco promissores para o controle de ninfas de $Q$. gigas em paricá. A reinfestação da área (Lunz et al., 2010) e a sobreposição de gerações (Maccagnan \& Martinelli, 2004) explica a continuidade de crescimento populacional das ninfas a despeito da aplicação dos inseticidas, embora menores em relação ao tratamento controle.

A verificação da persistência e da eficácia das moléculas, ou seja, do poder residual dos inseticidas no solo, ao longo dos meses seguintes à sua aplicação, é importante para culturas perenes como o cultivo do paricá, dado o ciclo de corte da espécie (cinco a sete anos, dependendo das condições de sítio e tratos silviculturais adequados), o que pode representar uma menor necessidade de aplicações ao longo do desenvolvimento das árvores e, consequentemente, menos gastos com aquisição e aplicação de inseticidas. 

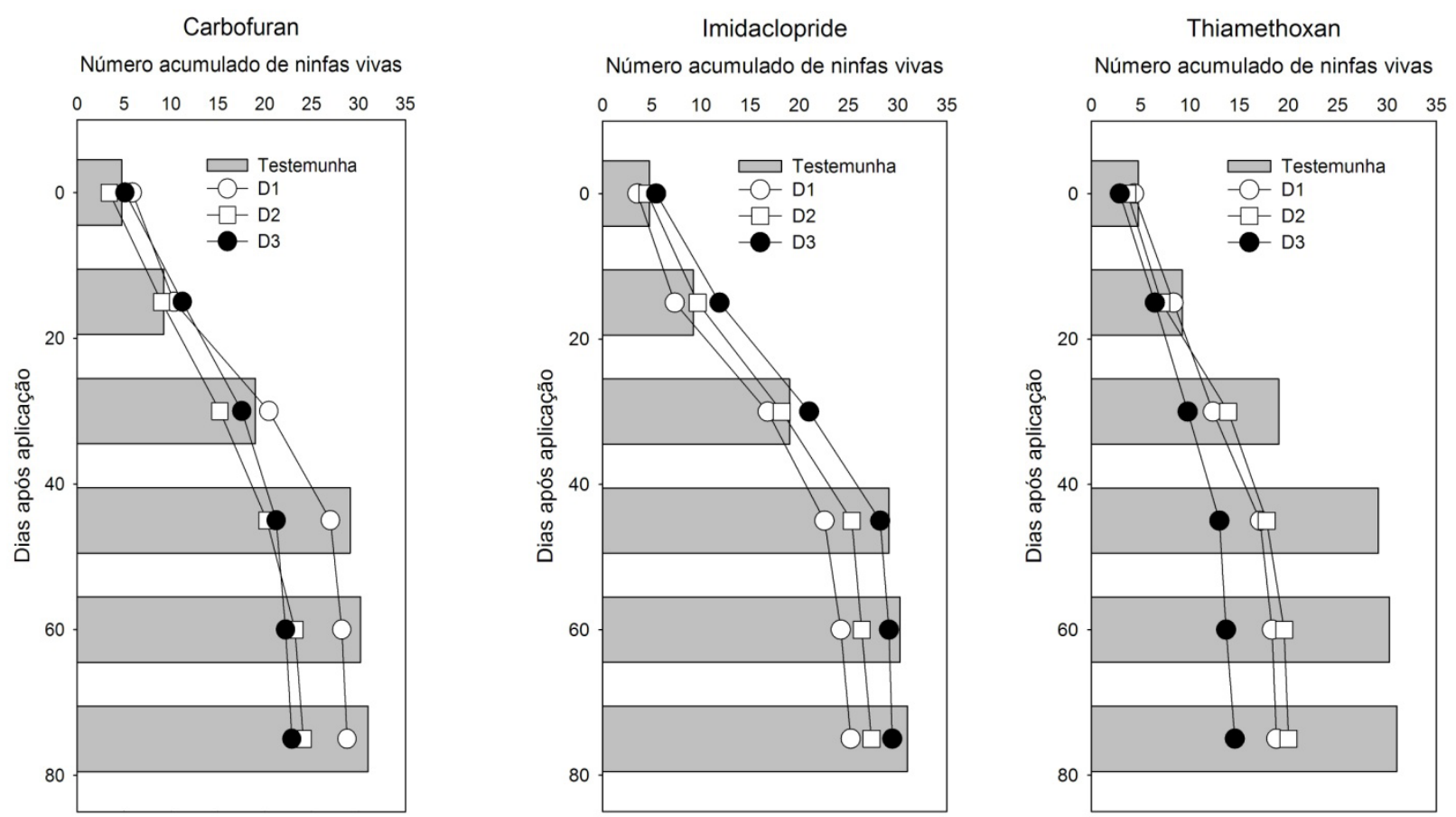

Figura 1. Alteração proporcional quinzenal no número de ninfas vivas de Quesada gigas em plantio de paricá presentes em relação ao número inicial em três dosagens de inseticidas (carbofurano: D1 = 4,0 L ha ${ }^{-1}$; D2 $=6,0 \mathrm{~L} \mathrm{ha}^{-1} \mathrm{e} \mathrm{D} 3=8,0 \mathrm{~L}^{-1}$; tiametoxam: D1 = 1,0 Kg ha-1; D2 = 1,5 $\mathrm{Kg} \mathrm{ha}^{-1}$ e D3 = 2,0 $\mathrm{Kg} \mathrm{ha}^{-1}$; imidacloprid: D1 = 2,5 L ha-1; D2 = 3,75 L ha-1 e D3 = 5,0 L ha' ${ }^{-1}$ ). Dom Eliseu, PA. 2010.

Os primeiros sinais da ação dos inseticidas testados foram observados aos 30 dias após a sua aplicação, mais notavelmente para o tiametoxam. Ao término do experimento, a maior eficiência foi verificada para todas as dosagens de tiametoxam (Tabela 1), sendo a menor delas $\left(1 \mathrm{~kg} \mathrm{ha}^{-1}\right)$ considerada a mais eficaz, por proporcionar igual eficiência que as demais (1,5 $\mathrm{kg} \mathrm{ha}^{-1}$ e $\left.2 \mathrm{~kg} \mathrm{ha}^{-1}\right)$ com uso de menor quantidade de produto, além de ser toxicologicamente menos impactante.

Tabela 1. Médias do número acumulado de ninfas vivas de Quesada gigas por trincheira em avaliações quinzenais até 75 dias após a aplicação de três inseticidas em três dosagens, em área experimental com plantio de paricá. Dom Eliseu, PA. 2010.

\begin{tabular}{|c|c|c|c|c|c|c|}
\hline \multirow{3}{*}{ Dose } & \multicolumn{6}{|c|}{ Dias após a aplicação dos produtos } \\
\hline & 0 & 15 & 30 & 45 & 60 & 75 \\
\hline & \multicolumn{6}{|c|}{ Carbofurano } \\
\hline D1 & $5,89 \mathrm{a}$ & $10,33 \mathrm{a}$ & $20,44 a b$ & $27,00 \mathrm{ab}$ & $28,22 \mathrm{ab}$ & $28,78 \mathrm{ab}$ \\
\hline D2 & $3,44 \mathrm{a}$ & $9,00 \mathrm{a}$ & $15,22 \mathrm{abc}$ & $20,22 \mathrm{abcd}$ & $23,22 \mathrm{abc}$ & $24,11 \mathrm{abc}$ \\
\hline \multirow[t]{2}{*}{ D3 } & $5,11 \mathrm{a}$ & $11,22 \mathrm{a}$ & $17,56 \mathrm{abc}$ & $21,22 \mathrm{abcd}$ & $22,22 \mathrm{abcd}$ & $22,89 \mathrm{abcd}$ \\
\hline & \multicolumn{6}{|c|}{ Imidacloprida } \\
\hline D1 & $3,56 \mathrm{a}$ & $7,33 \mathrm{a}$ & $16,78 \mathrm{abc}$ & $22,56 \mathrm{abc}$ & $24,22 \mathrm{abc}$ & $25,22 \mathrm{abc}$ \\
\hline D2 & $4,56 \mathrm{a}$ & $9,67 \mathrm{a}$ & $18,22 \mathrm{ab}$ & $25,33 \mathrm{abc}$ & $26,33 \mathrm{abc}$ & $27,33 \mathrm{abc}$ \\
\hline \multirow[t]{2}{*}{ D3 } & $5,44 \mathrm{a}$ & $11,89 \mathrm{a}$ & $21,00 \mathrm{a}$ & $28,22 \mathrm{a}$ & $29,11 \mathrm{ab}$ & $29,44 \mathrm{ab}$ \\
\hline & \multicolumn{6}{|c|}{ Tiametoxam } \\
\hline D1 & $4,33 \mathrm{a}$ & $8,33 \mathrm{a}$ & $12,33 \mathrm{bc}$ & $17,11 \mathrm{~cd}$ & $18,33 \mathrm{~cd}$ & $18,78 \mathrm{~cd}$ \\
\hline D2 & $3,67 \mathrm{a}$ & $7,11 \mathrm{a}$ & $13,89 \mathrm{abc}$ & $17,78 \mathrm{bcd}$ & $19,56 \mathrm{bcd}$ & $20,00 \mathrm{bcd}$ \\
\hline D3 & $2,89 \mathrm{a}$ & $6,44 \mathrm{a}$ & $9,78 \mathrm{c}$ & $13,00 \mathrm{~d}$ & $13,67 \mathrm{~d}$ & $14,56 \mathrm{~d}$ \\
\hline Controle & $4,78 \mathrm{a}$ & $9,22 \mathrm{a}$ & $19,00 \mathrm{ab}$ & $29,11 \mathrm{a}$ & $30,22 \mathrm{a}$ & $31,00 \mathrm{a}$ \\
\hline
\end{tabular}

Médias seguidas pelas mesmas letras, nas colunas, não diferem entre si pelo teste de Duncan, a 5\% de probabilidade. Carbofurano (Ralzer 350 TS): $\mathrm{D} 1=4,0 \mathrm{~L} \mathrm{ha}^{-1} ; \mathrm{D} 2=6,0 \mathrm{~L} \mathrm{ha}^{-1} \mathrm{e} \mathrm{D} 3=8,0 \mathrm{~L} \mathrm{ha}^{-1}$; tiametoxam (Actara $250 \mathrm{WG}$ ): D1 = 1,0 kg ha ${ }^{-1}$; 2 = 1,5 $\mathrm{kg} \mathrm{ha}^{-1} \mathrm{e} \mathrm{D} 3=2,0 \mathrm{~kg}^{-1}$; imidacloprida (Provado $200 \mathrm{SC}): \mathrm{D} 1=2,5 \mathrm{~L} \mathrm{ha}^{-1} ; \mathrm{D} 2=3,75 \mathrm{~L} \mathrm{ha}^{-1}$ e D3 $=5,0 \mathrm{~L} \mathrm{ha}^{-1}$. 
A eficácia de inseticidas à base de carbofurano e tiametoxam no controle de cigarras foi comprovada em cafezais (Reis \& Souza, 2007a, 2007b) e em reflorestamentos com paricá (Lunz et al., 2010). Contudo, o refinamento das dosagens de ambos os inseticidas permitiu observar que ótimos resultados para controle químico de ninfas de Q. gigas foram obtidos com uso de $50 \%$ da dosagem máxima de produtos à base de tiametoxam utilizada em plantios de café para controle de cigarras. Porém, mais estudos precisam ser realizados para comprovação destes resultados em outras condições ambientais, bem como estudar as causas dessa possível redução de dosagens de inseticidas, que é importante por permitir menores investimentos para aplicação de inseticidas em grandes áreas, considerando as perspectivas de expansão das áreas reflorestadas com paricá, gerando menor impacto ambiental.

\section{Agradecimentos}

Ao CNPq, pela concessão de bolsa DTI-3 ( ${ }^{\circ}$ 556763/2009-2) ao primeiro autor; à Rio Concrem Industrial Ltda., pela autorização de uso da área.

\section{Referências}

ANUÁRIO Estatístico da ABRAF 2013: ano base 2012. Brasília, DF: ABRAF, 2013. 148 p.

CARVALHO, P. E. R. Espécies arbóreas brasileiras. Brasília, DF: Embrapa Informação Tecnológica; Colombo: Embrapa Florestas, 2006. v. 2. 627 p.

CARVALHO, P. E. R. Paricá - Schizolobium amazonicum. Colombo: Embrapa Florestas, 2007. 8 p. (Embrapa Florestas. Circular técnica, 142).
IWAKIRI, S.; ZELLER, F.; PINTO, J. A.; RAMIREZ, M. G. L.; SOUZA, M. M.; SEIXAS, R. Avaliação do potencial de utilização da madeira de Schizolobium amazonicum "Paricá" e Cecropia hololeuca "Embaúba" para produção de painéis aglomerados. Acta Amazonica, Manaus, v. 40, p. 303-308, 2010.

LUNZ, A. M.; AZEVEDO, R. de; MOURÃO JUNIOR, M.; MONTEIRO, O. M.; LECHINOSKI, A.; ZANETI, L. Z. Método para monitoramento de ninfas de cigarras e controle com inseticidas em reflorestamentos com paricá. Pesquisa Agropecuária Brasileira, Brasília, DF, v. 45, p. 631-637, 2010.

LUNZ, A. M.; AZEVEDO, R. de; MOURÃO JUNIOR, M.; MONTEIRO, O. M. Recomendações para o monitoramento de cigarras [Quesada gigas (Olivier), Hemiptera: Cicadidae] em reflorestamentos com paricá [Schizolobium parahyba var. amazonicum (Huber ex Ducke) Barneby]. Belém: Embrapa Amazônia Oriental, 2012. 12 p. (Embrapa Amazônia Oriental. Circular técnica, 46).

MACCAGNAN, D. H. B.; MARTINELLI, N. M. Descrição das ninfas de Quesada gigas (Olivier) (Hemiptera: Cicadidae) associadas ao cafeeiro. Neotropical Entomology, Londrina, v. 33, p. 439-446, 2004.

MARTINELLI, N. M.; ZUCCHI, R. A. Cigarras (Hemiptera: Cicadidae: Tibicinidae) associadas ao cafeeiro: distribuição, hospedeiros e chave para as espécies. Anais da Sociedade Entomológica Brasileira, Londrina, v. 26, p. 133-143, 1997.

REIS, P. R.; SOUZA, J. C. Controle associado. Cultivar, Pelotas, n. 97, p. 33-36, 2007a.

REIS, P. R.; SOUZA, J. C. Sugadora de café. Cultivar, Pelotas, n. 102, p. 6-7, 2007b.

ZANUNCIO, J. C.; PEREIRA, F. F.; ZANUNCIO, T. V.; MARTINELLI, N. M.; PINON, T. B. M.; GUIMARÃES, E. M. Occurrence of Quesada gigas on Schizolobium amazonicum trees in Maranhão and Pará states, Brazil. Pesquisa Agropecuária Brasileira, Brasília, DF, v. 39, p. 943-945, 2004. 